\title{
VLBI OBSERVATIONS OF THE RADIO JET IN 3C 273
}

\author{
S. C. Unwin \\ California Institute of Technology, Pasadena, U.S.A.
}

\section{R. J. Davis}

Nuffield Radio Astronomy Laboratories, Jodrell Bank, U.K.

\begin{abstract}
We present a new high dynamic range map of the quasar 3C 273, made from observations with a VLBI network of 12 telescopes. This new map at $18 \mathrm{~cm}$ wavelength has one of the highest dynamic ranges yet achieved with VLBI, and it shows the 'jet' extending to at least 180 milliarcsec, or $330 \mathrm{pc}$ from the nucleus of the quasar. Strong limits can be placed on the brightness of any 'counter-jet' on kiloparsec-scales, as no emission is visible on the opposite side of the 'core'. Combining with other VLBI, VLA and MERLIN maps shows that the jet is visible and continuous over a very large range of scales, from $1 \mathrm{pc}$ to $40 \mathrm{kpc}$.
\end{abstract}

\section{VLBI MAP}

The new map, shown in Figure 1, was made using the Jodrell Bank OLAF mapping software (R.G. Noble, A. Bailey and T.W.B. Muxlow, unpublished), which corrects for errors in the data due to $(a)$ station-dependent amplitudes and phases, and $(b)$ baselinedependent terms. Station corrections are part of normal 'hybrid' mapping, but for high SNR data, as in this case, the map quality is limited by baseline errors, and correcting them makes a dramatic improvement.

Using this data we have achieved one of the highest dynamic ranges of any VLBI map made to date, over 7000:1 (defined as peak brightness to rms noise level). Away from the map center, the noise level is $1.2 \mathrm{mJy} /$ beam, rising to $1.0 \mathrm{mJy} /$ beam at the largest feature believed to be an artifact, just east of the core. Even this feature is only $0.1 \%$ of the peak brightness. Although the resolution is poorer in the $\mathrm{N}-\mathrm{S}$ direction, the jet is unresolved on our map at all points along its length.

'Superluminal' components seen on VLBI maps at higher frequencies and higher resolution (e.g., Unwin et al. 1985) are identified as 'knots' on our map out to about 20 milliarcsec from the core, and the slight jet curvature is consistent with the positions of those features. There are indications the jet extends beyond the area plotted, but shorter baselines and better self-calibration are needed to map this weak emission reliably.

Some components further down the jet are sufficiently well-defined that proper motions could be detected between them in a few years. We therefore plan to repeat the observation within the next year or so, to search for differences between the two maps. With a map of similar quality, detection of proper motions similar to those seen in the close-in components will be possible. A direct measurement at larger radii would be a critical test of the Davis, Muxlow, and Conway (1986) model of the jet morphology, which seeks to explain the wiggles seen in the ridge-line of the jet in terms of precession of the jet axis. 


\section{DISCUSSION}

The brightness of the jet along the ridge line decays rapidly with distance from the nucleus, in a power-law fashion, out to the first large-scale knot at 7 arcsec. A smooth relation over three orders of magnitude in radius suggests that the physical parameters of the jet also follow simple power laws, as found for 3C 120 by Walker, Benson, and Unwin (1987).

One possible model which reproduces the main features of the source over a wide range of scales is one in which the radio emission close to the core is caused by internal turbulence, or shocks, in a flow which moves out rapidly. The decay in jet brightness is caused by loss of energy by radiation, leaving power only in the bulk flow. Further out, the jet gradually slows down, and becomes more visible again, by interaction with the confining external medium. This model contrasts somewhat with the 'standard' model, in that the jet is moving slowly by the time it reaches the hotspot at the end of the head.

No 'counter-jet' is seen on the opposite side of the core at any resolution in our new data, or indeed, of any other observation of this object in any waveband. Our map yields a limit of several 100:1 on the jet:counter-jet ratio on scales up to about 0.1 arcsec. Since the outer 'head' appears to be genuinely one-sided, and is fed with energetic particles by the jet, it seems that $3 C 273$ represents the best case yet for an intrinsically one-sided jet.

These observations were conducted by the US VLBI Network Users Group, and we thank all those who help make global VLBI possible. This research was supported by the NSF, via grant 85-09822 to the Owens Valley Radio Observatory.

\section{REFERENCES}

Unwin, S.C., Cohen, M.H., Biretta, J.A., Pearson, T.J., Seielstad, G.A., Walker, R.C., Simon, R.S., and Linfield, R.P.: 1985, Astrophys. J. 289, 109.

Davis, R.J., Muxlow, T.W.B., and Conway, R.G.: 1985, Nature 318, 343.

Walker, R.C., Benson, J.M., and Unwin, S.C.: 1987, Astrophys. J. 316, 546.

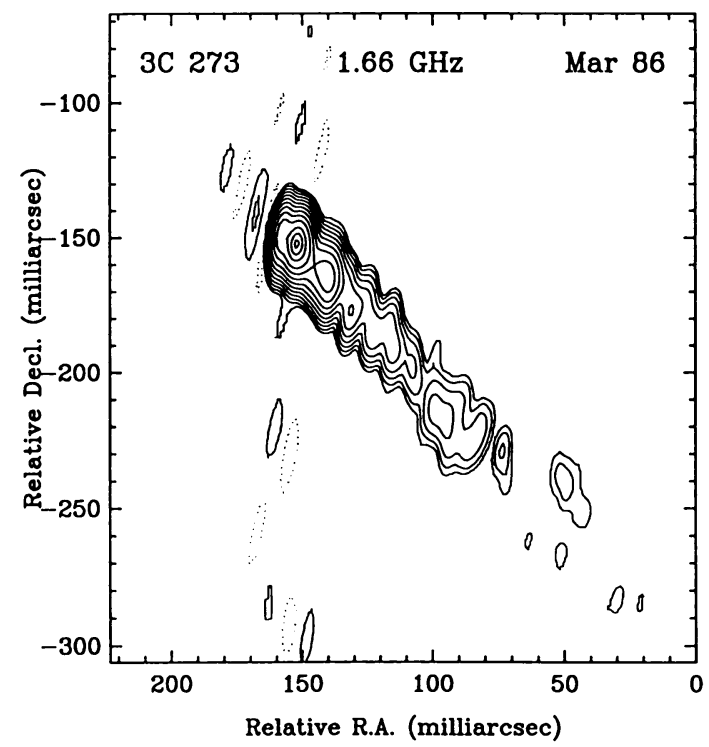

FIGURE 1. VLBI map of $3 \mathrm{C} 273$ at $18 \mathrm{~cm}$ wavelength. The restoring beam is $12 \times 4$ milliarcsec, and contours are drawn at $-0.05,0.05,0.1,0.2,0.4,0.8,1.6,3.2,6.4,13,25$, 50,75 , and $90 \%$ of the peak (negative contours dashed). 